\title{
MIT0-7-a weekly regimen associated with a better quality of life for patients with advanced ovarian cancer
}

$\mathrm{O}$

varian cancer is diagnosed in over 200,000 women each year worldwide, with 125,000 deaths.

These figures confirm that ovarian cancer is the most lethal of the gynaecological malignancies, mainly owing to the fact that more than $70 \%$ of women with this cancer are diagnosed with advanced disease. The standard treatment approach for patients with advanced-stage ovarian cancer is surgery followed by combination chemotherapy with carboplatin plus paclitaxel administered every 3 weeks. However, this regimen is toxic and can cause alopecia, neurotoxicity and fatigue, which have a negative impact on patients quality of life (QOL). The results of the MITO-7 phase III trial, published in The Lancet Oncology, suggest that a weekly chemotherapy regimen of carboplatin plus paclitaxel-associated with a lower toxicity profile and an improved QOL-represents an alternative first-line treatment for women with advanced-stage disease.

Previous phase II studies had suggested that a weekly schedule of paclitaxel can reduce the toxicity in patients by decreasing the peak levels of the drug in the plasma. Therefore, "in 2008, our hypothesis was that the experimental, weekly schedule [of carboplatin plus paclitaxel] could be associated with a better QOL compared with the standard treatment", explains Sandro Pignata, lead author of the study.

The MITO-7 trial analysed 810 patients, across 67 institutions in Italy and France, between November 2008 and March 2012. Specifically, 404 patients were randomly assigned to receive the standard carboplatin plus paclitaxel regimen every 3 weeks, whereas 406 patients were assigned to the weekly regimen. Of note, the two arms differed only in the regimen schedule, as the doses of the two drugs were adjusted to guarantee a similar dose intensity. Initially, the study was designed to have only one primary end point-health-related QOL, as measured by the Functional Assessment of Cancer Therapy for Ovarian Cancer (FACT-O) trial outcome index score. However, the results of the Japanese NOVEL trial showing that-compared with the standard 3-weekly regimen of paclitaxel and carboplatin-a weekly regimen of dose-dense paclitaxel with carboplatin administered every 3 weeks, produced a substantial improvement in progressionfree survival (PFS), prompted the authors to add PFS as co-primary end point.

The MITO-7 trial did not show any significant difference in PFS and a similar efficacy was observed in the two arms. Notably, the MITO-7 and NOVEL trials had different experimental schedules, including different doses of paclitaxel, different schedule of carboplatin and different patient ethnicity. However, as co-author Massimo Di Maio emphasizes,

"the weekly schedule produced significantly less toxicity (particularly less hair loss and neurotoxicity) and was associated with a significantly better QOL”. This was an interesting result as the NOVEL trial had reported an increase in toxicity with the weekly regimen. Di Maio continues, "in detail, the standard treatment produced a significant worsening of QOL after each cycle of administration, whilst the QOL was much less affected by the weekly schedule," in which FACT-O scores stabilized after a transient worsening at week 1 . An analysis of the adverse events showed that patients assigned to the weekly regimen compared with women receiving treatment every 3 weeks, had a more-favourable pattern for neutropenia (grade 3-4, 42\% [weekly] versus 50\% [every 3 weeks]), thrombocytopenia (grade $3-4,1 \%$ versus $7 \%$ ), alopecia (grade 2, $29 \%$ versus 59\%) and neuropathy (grade $\geq 2.6 \%$ versus $17 \%$ ).

"From a patient point-of-view, we think that the results obtained with the weekly schedule in terms of toxicity and QOL

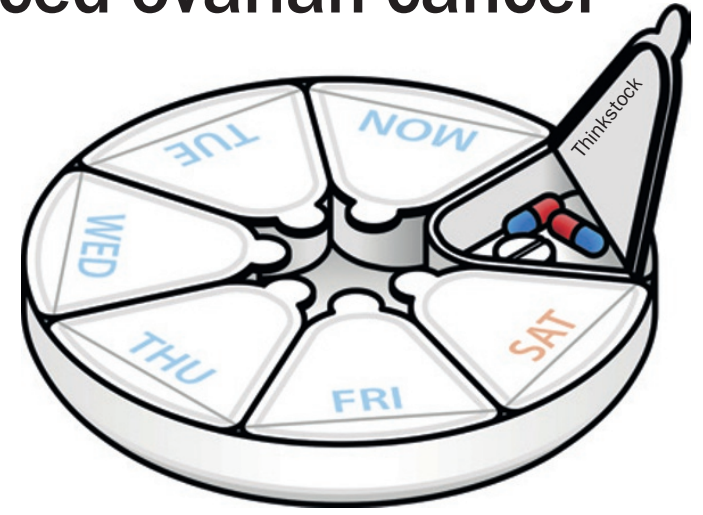

are of great clinical value," comments co-author Francesco Perrone. Notably, despite a lower toxicity, the weekly regimen is quite challenging for patients, as indicated by a slightly better adherence to planned treatment in the standard every-3-week chemotherapy arm.

In addition to the MITO-7 trial, two ongoing trials, GOG-262 (NCT01167712) and ICON-8 (NCT01654146), are testing the efficacy of alternative weekly regimen in women with ovarian cancer. Once the results of these studies are available, "a weekly schedule might be considered among the possible first-line treatment options for advanced-stage ovarian cancer in clinical practice," says Pignata. Collectively, the researchers agree that "for future research, considering the favourable tolerability and QOL profile in our trial, the MITO-7 weekly schedule could represent an ideal chemotherapy backbone for the combination with biologic agents". The results of the MITO-7 trials are, therefore, not only a step ahead at improving the QOL of patients, but also a platform for developing new, safer, and more-effective treatment strategies for this deadly disease.

Alessia Errico

Original article Pignata, S. et al. Carboplatin plus paclitaxel once a week versus every 3 weeks in patients with advanced ovarian cancer (MITO-7): a randomised, multicentre, open-label, phase 3 trial. Lancet Oncol. doi:10.1016/S1470-2045(14)70049-X 